\title{
AN INVESTIGATION OF FALSE POSITIVE DOSIMETRY RESULTS
}

\author{
M.A. Lewandowski. S. A. Davis. T. E. Goff, and C. F. Wu \\ Waste Isolation Division. Westinghouse Electric Corporation. \\ Carlsbad. New Mexico. USA
}

\begin{abstract}
The Waste Isolation Pilot Plant (WIPP) is a facility designed for the demonstration of the safe disposal of transuranic waste. Currently. the radiation source term is confined to sealed calibration and check sources since WIPP has not received waste for disposal. For several years the WIPP Dosimetry Group has operated a Harshaw Model 8800C reader to analyze Harshaw 8801-7776 thermoluminescent cards (3 TLD-700 and 1 TLD-600) with 8805 holder. The frequency of false positive results for quarterly dosimeter exchanges is higher than desired by the Dosimetry Group management. Initial observations suggested that exposure to intense ambient sunlight may be responsible for the majority of the false positive readings for element 3 . A study was designed to investigate the possibility of light leaking through the holder and inducing a signal in element 3 . This paper discusses the methods and results obtained, with special emphasis placed on recommendations to reduce the frequency of light-induced false positive readings. (This research is supported by the U.S. Department of Energy under Contract DE-AC04-86AL31950.)

\section{Introduction}

The WIPP Dosimetry Group is currently experiencing a false positive rate of approximately 10 percent of its thermoluminescent dosimeter (TLD) results. These false positives are generally in the range of 100 to $400 \mathrm{mrem}$. indicating that the problem is not a statistically acceptable function of background subtraction. It has been observed that the rate of occurrence is increasing (Figure 1). Currently, the radiation doses can be discounted, because only small check sources are on site. With wastes expected in November 1997, significant efforts are being made to resolve the problem. This investigation focuses on light leakage effects on the teflon and TLD crystals. This includes leakage through hanger materials and the identification label window. Based on this investigation, it appears that even incandescent light may cause spurious responses; further research will be conducted on this hypothesis.
\end{abstract}

The WIPP facility is designed as the repository for transuranic radioactive wastes from the United States nuclear weapons program. Transuranic waste will be received from weapons complex facilities throughout the United States. The waste will be shipped in Department of Transportation (DOT) category type B containers, called TRUPACT-Ils. which have been approved by the Nuclear Regulatory Commission for transuranic waste. Each TRUPACT-II can hold 1455 -gallon drums ( 55 gallons each), or two waste boxes. The contents of the TRUPACT-IIs are unloaded on the surface and the contents transported 2,150 feet underground. into rooms mined out of rock salt. Any exposure of personnel to radiation will primarily occur during the unloading and handling of the waste.

Department of Energy laboratory accreditation is necessary in many categories. because of the highly variable radiation fields that can be expected from the waste. which will arrive at the site from many locations across the country. Although most of the activity from the waste that will be disposed consists of transuranic isotopes, the external radiation fields are from higher energy photon sources, primarily fission products. Because of the fission products, the dosimeters must be capable of detecting beta radiations in case a spill of the waste occurs. Neutron detection is also necessary, because the waste may contain californium-252, or mixtures of the transuranic alpha emitters and low atomic number materials from some facilities.

The Harshaw 8801 personnel dosimeter is used and processed using a Harshaw 8800 TLD reader. ${ }^{1}$ The 8800 reader uses hot nitrogen gas as the heating mechanism. This provides very even heating and easily quantified temperatures of the gas.: The TLD card is enclosed in a plastic hanger, with lead and copper shields, a thin mylar beta "window" and an identification slot. Identification information, such as name, storage rack location, and/or "assign by" date is inserted 


\section{DISCLAMMER}

Portions of this document may be illegible in electronic image products. Images are produced from the best available original document. 
This document has been reproduced directly from the best possible copy.

It is available to DOE and DOE contractors at the following addresses:

Office of Scientific and Technical Information

P.O. Box 62

Oak Ridge, TN 37831

Prices available from (615) 576-8401

Available to the public from the National Technical Information Services

U. S. Department of Commerce

5285 Port Royal Road

Springfield, VA 22161

Processing and final preparation of this report was performed by the Waste Isolation Pilot Plant Management and Operating Contractor for the U.S. Department of Energy under Contract No. DE-ACO4-86AL31950. 
into the identification slot while the hanger is open. The 8801 TLD consists of four crystal elements held in place with a teflon film. Teflon is a tetrafluoroethylene resin plastic manufactured by DuPont. Teflon is used due to its ability to withstand the high temperatures $\left(300^{\circ}\right)$ that occur during the reading of the dosimeters. The four TLD elements are lithium fluoride. with one element enriched in Li- 6 and three enriched in Li-7. The Li-6 element is very neutron sensitive. and the Li-7 chips show virtually no response to neutrons. ${ }^{2}$ One of the $\mathrm{Li}-7$ chips is very thin $(0.15 \mathrm{~mm})$ for beta indication. This chip is placed behind a thin mylar film to reduce low energy beta attenuation. This chip is responsible for most of the false positives that occur at WIPP.

The WIPPs dosimerry system has been encountering an increasing level of positive readings over the last four years (Figure 1). These positive readings are generally considered false because there are no significant sources on site. and the on-site sources are not available for exposure to the personnel incurring positive readings. Personnel who work with the radiation check sources and have positive TLD readings are assigned the doses.

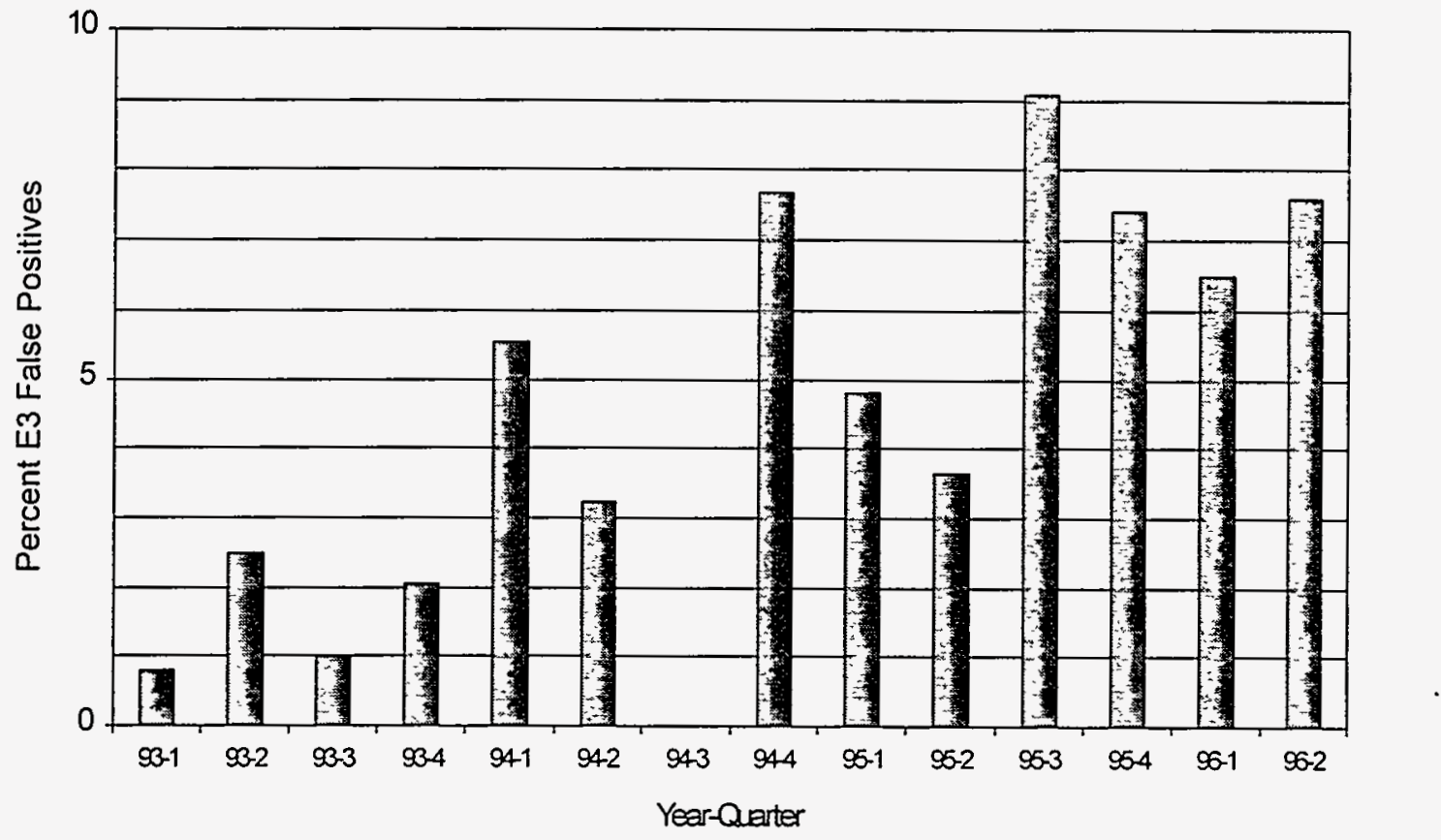

Figure 1. Percentage of element 3 positive readings for the period of 1993 to 1996.

Declaring positive readings as false places the entire dosimetry program in question. If positive readings are said to be inaccurate, some observers may consider the possibility that true doses may be incorrectly determined to be negative (i.e., false negatives). Although false positive readings occur at many facilities. inaccurate readings at WIPP will have a very high visibility. Because of the very low doses expected at WIPP. false positives at this facility will have severe impact on ALARA goals and administrative limits. 


\section{Problem Analysis and Handling}

The lower limit of detection of the dosimetry system has been calculated as 10 mrem deep dose. and 35 mrem shallow dose. These values are based on a study of background accumulation on thousands of TLDs issued in 1989. Based on the calculations. a maximum 5 percent false positive rate would be expected, due to statistical variation in background. Not only is the false positive rate consistently greater than 5 percent, the high numerical values are clearly not just from statistical background variations.

Currently, several approaches are being taken to handle false positive dosimeter readings. In some cases. the shielded deep-dose chip is the highest reading. and all other elements are less than their lower limit of detection. In this case. the "element I" reading can be considered a false positive reading. because no radiation fields could result in this elementresponse relationship. Based on this, the dose for the individual can be considered zero.

Area monitoring TLDs, especially those in outside areas, have a particular vulnerability to false positives. An additional TLD was placed at each monitoring location to record a dose value in the event that one of the dosimeters has a false positive, or unusually high reading. If the glow curve for a high element reading is abnormal, then the TLD is not used, and the dose for that position is based on the other dosimeter.

WIPP Dosimetry uses two methods of glow curve refitting. The primary methodology is software developed by Dosimeter Services Company. This program normalizes the abnormal glow curve to a well-shaped glow curve from another element of the same badge. The area of the normalized glow curve is then used to determine the correct response for the TLD element. The normalized response is then used for a manual calculation of the dose, using the standard algorithm. WIPP Dosimetry has also developed an in-house methodology to refit glow curves. The WIPP system uses glow curves of different TLDs. Although different TLDs are used. the same element is used for comparison. TLDs are also selected to have similar fade periods. In this way, a thin chip element can be refit to another thin chip glow curve.

In cases where the glow curve refit techniques do not result in clearing the false positive reading, the dose can be set to zero, based on a lack of radiation source term. Only a small group of workers at WIPP, radiological control personnel. have access to the radioactive check sources. If the radiological control personnel have positive readings on their dosimeters, and the glow curve refitting or high shielded element methodologies are not successful, these personnel are then assigned the positive reading. For others, the doses can be assumed as zero.

All these techniques will be of questionable value, however, when radioactive wastes begin arriving at the WIPP site. It will be difficult to satisfy personnel that their doses should be changed when they are aware that radioactive materials are stored or being transported on site.

Several potential conditions could cause the false positive readings on TLDs. Dirt. grease, and hand oils or powders could contaminate the chips and result in luminescence at the high temperatures encountered during the readings of the dosimeters. While the luminescence could show up outside the normal glow curve area, it could also add to the response inside the normal glow area.

Currently, cards with false positives are not removed from service. The cards generally are not tested, and there has been no research to determine if the false positives recur from these cards over a period of time. The dose history of cards indicating false positives is unknown. A high dose history could possibly also result in false positive readings. This data is not readily available, but could be generated from the historical readings database.

Generally, false positives are considered to be caused by light leaks and the subsequent response of teflon film and TLD crystals. A normal, long fade time glow curve from a significant exposure consists of one symmetrical narrow peak. If the curve is contaminated with non-radiation-induced response. then the glow curve can be wider, and consist of more 
than one peak. or have buildup before and after the normal peak location. Poor glow curves are depicted with the image of the element 1 and 3 glows from card 8162 (Figure 2). Most false positive responses of TLDs at WIPP have bad glow curves.

A study conducted by Oak Ridge National Laboratory (ORNL) indicates that most of the light response comes from the teflon rather than from the TLD crystals. The response correction of the element 3 chip is larger than for the other chips because of the smaller mass of the thin chip. Coupled with the greater possibility of light leakage through the beta window, this results in a significant potential for over-response of this element.

\section{Experiments}

A study was conducted to determine the source of light leakage that could expose the elements and mylar of the TLD. TLDs were annealed, and five TLDs (per each condition) were placed in varying conditions for exposure to sunlight. TLDs were placed in normal holders, holders with a new mylar beta window, holders with poor/damaged mylar, and holders with electrical tape over the beta window.

The hangers were then exposed to sunlight while hanging on a fence for one week. At the end of the week. the TLDs were read. While the small number of TLDs made a quantitative evaluation difficult. it appeared that new mylar is as effective in blocking ambient sunlight as the black electrical tape.

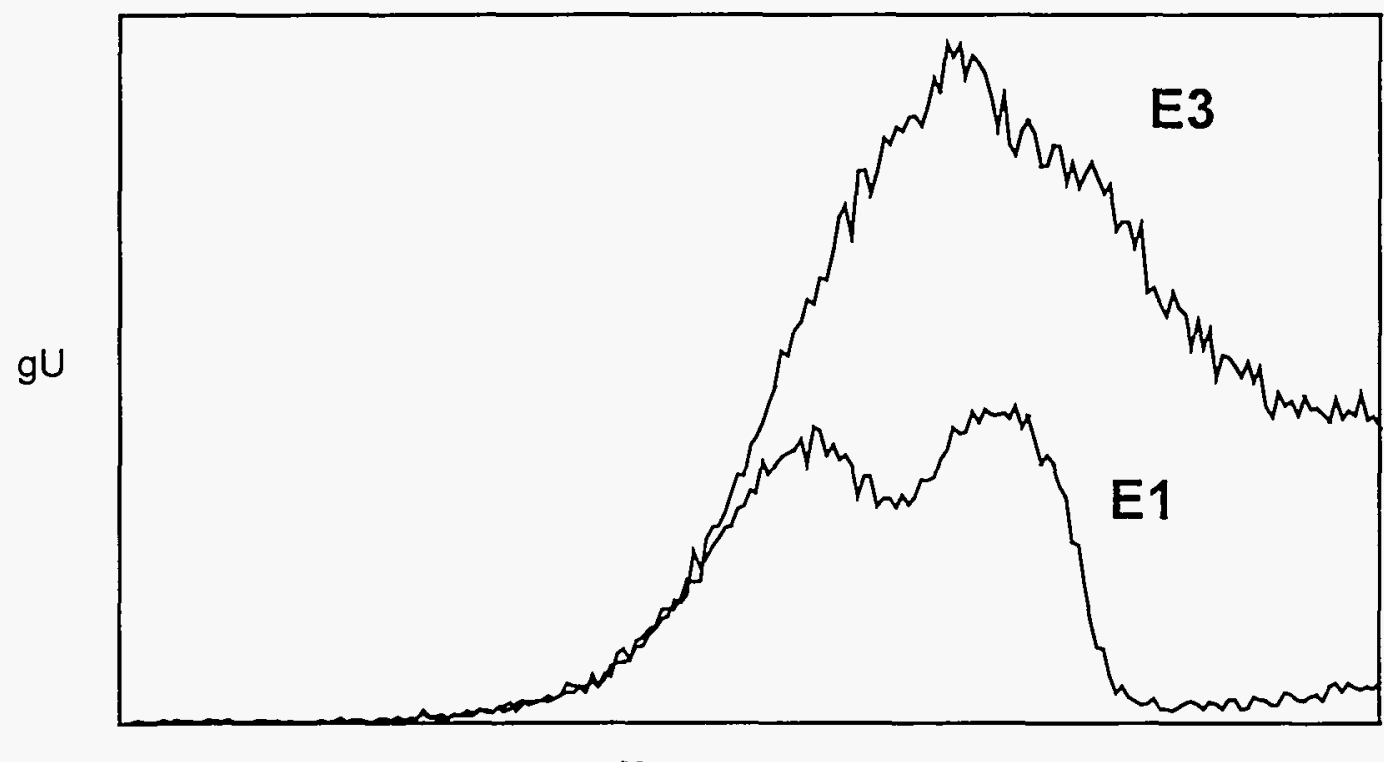

Temperature

Figure 2. Glow Cures of Elements 1 and 3 of TLD card 8162.

Another study, with a larger number of dosimeters, was also conducted. The study was intended to quantify the impacts of two variables: new versus normal mylar, and light leakage through the identification slot. Twenty TLDs were exposed under varying holder arrangements: (1) normal badges, (2) normal mylar windows. (3) new mylar windows, (4) tape over normal mylar, and (5) tape over new mylar. In addition, seven cards not in holders were exposed to ambient incandescent light in the TLD laboratory. and 40 control dosimeters were not exposed to light. 
It is found that all results are very close together. with the exception of the ambient light exposure. This produced one of the most striking observations of the study: laboratory lighting may cause a response in TLD cards. This was a surprise. because the laboratory has historically been considered safe for exposure of the bare TLD cards. The laboratory is equipped with incandescent lights. which were thought to prevent the induction of the teflon response.

\section{Discussion}

Based on the study, two conclusions may be reached: (1) ambient lighting in the TLD laboratory may contribute to false positives. and (2) the identification slot may be a source of leakage.

Several options are being considered in the attempt to resolve the false positive issue at WIPP. These options are:

1. Incorporate at least a one-week delay between initial and preissue anneal of TLDs. Any TLD returning a high response would not be assigned for personnel monitoring. This would catch dosimeters that build up a response in a short period of time.

2. Remove cards that have a history of false positives from service. Newly installed TLD reader control computer software allows the easy location of dosimeters. without the tedious manual inspection of number code labels. This reduces the possibility that repeat false positive potential cards could be reissued

3. Incorporate controls of TLDs to laboratory lighting. This would include keeping TLDs stored in the laboratory covered, minimizing the time TLD cards are exposed to the light during loading and unloading operations, and, covering the lights in the TLD laboratory with yellow tinted plastic. This was observed at another facility, and is mentioned in the ORNL study. ${ }^{3}$

4. Initiate cleanliness controls for card handling. This would require that cards be cleaned prior to issue, handled with cotton gloves, and cleaned prior to reading if there is any indication that dirt, oil, or other contaminants could have entered the holder.

Further studies are required to better define the generation of false positives. Harshaw has provided WIPP with a set of dosimeters with different TLD crystals and teflon materials. These cards need to be evaluated regarding their response to light and how the composition of various cards currently in stock is related to the materials in the new Harshaw cards. This may involve testing the current inventory for response to light.

WIPP Dosimetry utilizes Harshaw blue holders. Harshaw also manufactures black holders. Dosimetry will conduct an evaluation of the impact on false positives when the black holders are used. With the relatively small number of personnel being monitored, changing to a different holder would present a limited and acceptable cost. In some cases. the elements above the identification slot appear to have been exposed to light. One hypothesis is that this exposure is caused by photocopiers. Dosimetry will conduct tests to determine if exposure to photocopier light could cause false positive response. The exposure angle, the unusual light intensity, and the wavelength will be considered.

The reproducibility and annealability of the light-induced response will be evaluated. Of critical importance is the ability to determine if a card will generate a false positive under normal use conditions. Currently, the only check is the preissue anneal value. If a card has generated a false positive, a low reading shortly after an anneal may not be adequate evidence that the reading will be high after a long period of time due to trap shifting or other phenomena.

There is a need to determine if cards repeat false positives. This is most easily accomplished by a search of the dose database for dosimeters with recurring positive responses. This would determine if removal of cards with false positives would reduce the occurrence of false positives in the future. 
No evaluation has been conducted to determine if dose history contributes to false positives. While it is known that very high doses require removal of a card from service. WIPP has not evaluated the impact of a large number of doses (i.e.. from non-accident range testing) on card residual readings. This will also be accomplished with a database search.

\section{References}

1. WP 12-3, Dosimetry Program Manual. Waste Isolation Pilot Plant. Carlsbad. New Mexico. USA. 1997

2. Harshaw Model 8800 TLD Reader Users Manual. Harshaw-Bicron. Solon. Ohio. USA, 1996

3. The Effect of Visible Light on Harshaw Model 8801 Thermoluminescent Dosimeters: Oak Ridge National Laboratory, Oak Ridge, Tennessee. USA, 1990

Processing and final preparation of this report was performed by the Waste Isolation Pilot Plant Management and Operating Contractor for the U.S. Department of Energy under Contract No. DE-ACO4-86AL31950. 